INPLASY

PROTOCOL

To cite: Rong et al. The efficacy and safety of regimens for Helicobacter pylori eradication treatment in China: a systemic review and network meta-analysis. Inplasy protocol 2021110089. doi:

10.37766/inplasy2021.11.0089

Received: 24 November 2021

Published: 24 November 2021

Corresponding author:

Rong Lin

selinalin35@hotmail.com

Author Affiliation:

Takeda Pharmaceutical

Company

Support: Takeda

Pharmaceutical Company.

Review Stage at time of this submission: Data extraction.

Conflicts of interest:

None declared.

\section{The efficacy and safety of regimens for Helicobacter pylori eradication treatment in China: a systemic review and network meta-analysis}

Rong, L1; Li, J2; Shi, H³; Zhou, F4; Xie, L55.

Review question / Objective: To explore the effectiveness and safety of eradication therapies (including first- and rescue eradication therapy) for Chinese patients with $\mathrm{Hp}$ infection. Condition being studied: Helicobacter Pylori ( $\mathrm{Hp}$ ) infection is involved in the development of gastric malignancies, particularly gastric cancer and gastric mucosa-associated lymphoid tissue lymphoma. With the prevalence of antibiotic resistance in Helicobacter pylori $(\mathrm{Hp})$ globally, to identify a more effective treatment regimen on the eradication of $\mathrm{Hp}$ becomes high priority for clinicians. There are several some network meta-analyses investigating the effectiveness and safety of various regimens for $\mathrm{Hp}$ eradication treatment worldwide, however, the findings cannot reflect the certain circumstances in China.

INPLASY registration number: This protocol was registered with the International Platform of Registered Systematic Review and Meta-Analysis Protocols (INPLASY) on 24 November 2021 and was last updated on 24 November 2021 (registration number INPLASY2021110089).

\section{INTRODUCTION}

Review question / Objective: To explore the effectiveness and safety of eradication therapies (including first- and rescue eradication therapy) for Chinese patients with $\mathrm{Hp}$ infection.
Condition being studied: Helicobacter Pylori ( $\mathrm{Hp}$ ) infection is involved in the development of gastric malignancies, particularly gastric cancer and gastric mucosa-associated lymphoid tissue Iymphoma. With the prevalence of 
antibiotic resistance in Helicobacter pylori (Hp) globally, to identify a more effective treatment regimen on the eradication of $\mathrm{Hp}$ becomes high priority for clinicians. There are several some network meta-analyses investigating the effectiveness and safety of various regimens for $\mathrm{Hp}$ eradication treatment worldwide, however, the findings cannot reflect the certain circumstances in China.

\section{METHODS}

Search strategy: An electronic search was conducted in PubMed, EMBASE, the Cochrane Library, CNKI, Wanfang, and CBM on 24th August, 2021, without limitation on language, but with limitation on China. We included all the eligible RCTs from 2010 to 2021. The electronic search will be supplemented by hand-searching of reference lists of relevant systematic reviews at full-text screening stage. This is intended to identify any additional published or unpublished material (grey literature) not retrieved by the electronic search.

1. PubMed:

\#1."Helicobacter Infections"[Mesh] OR "Helicobacter pylori"[Mesh] OR Helicobacter[tiab] OR Campylobacter[tiab]. \#2. ("controlled clinical trial"[pt] OR "Controlled Clinical Trials as Topic"[MeSH] OR "Random Allocation"[MeSH] OR "Double-Blind Method"[MeSH] OR "singleblind method"[MeSH] OR "Control Groups"[MeSH] OR "cross-over studies"[MeSH] OR random*[tiab] OR placebo[tiab] OR trial[tiab] OR groups[tiab] OR crossover[tiab] OR cross-over[tiab]) NOT ("Animals " [ Mesh] NOT ("Humans"[Mesh] AND "Animals"[Mesh])). \#3. \#1 AND \#2.

\#4. "China"[Mesh] OR China OR Chinese OR Taiwan OR "Hong kong" OR Hongkong OR Macau OR Macao OR Beijing OR Shanghai OR Tianjin OR Chongqing OR "Inner Mongolia" OR Tibet OR Guangxi OR Sinkiang OR Ningxia OR Xinjiang OR Hebei OR Shanxi OR Liaoning OR Jilin OR Heilongjiang OR Jiangsu OR Zhejiang OR Anhui OR Fujian OR Jiangxi OR Shandong OR Henan OR Hubei OR Hunan OR Guangdong OR Hainan OR Sichuan OR
Guizhou OR Yunnan OR Shaanxi OR Gansu OR Qinghai.

\#5. \#3 and \#4.

2. EMBASE:

\#1. exp Helicobacter infection/ OR exp Helicobacter pylori/ OR Helicobacter.ab,ti,kw. OR Campylobacter.ab,ti,kw.

\#2. (exp controlled clinical trial/ OR exp "Controlled Clinical Trial (Topic)"/ OR double blind procedure/ OR control group/ OR crossover procedure/ OR single blind procedure/ OR triple blind procedure/ OR placebo/ OR exp randomization/ OR (random* OR trial OR groups OR placebo* OR crossover OR "cross-over").ab,ti,kw.) AND exp human/

\#3. \#1 AND \#2.

\#4. exp China/ OR (China OR Chinese OR Taiwan OR "Hong kong" OR Hongkong OR Macau OR Macao OR Beijing OR Shanghai OR Tianjin OR Chongqing OR "Inner Mongolia" OR Tibet OR Guangxi OR Sinkiang OR Ningxia OR Xinjiang OR Hebei OR Shanxi OR Liaoning OR Jilin OR Heilongjiang OR Jiangsu OR Zhejiang OR Anhui OR Fujian OR Jiangxi OR Shandong OR Henan OR Hubei OR Hunan OR Guangdong OR Hainan OR Sichuan OR Guizhou OR Yunnan OR Shaanxi OR Gansu OR Qinghai).ti,ab,kw,ad,cp.

\#5. \#3 AND \#4.

3. The Cochrane Library:

\#1 MeSH descriptor: [Helicobacter Infections] explode all trees.

\#2 MeSH descriptor: [Helicobacter pylori] explode all trees.

\#3 Helicobacter OR Campylobacter.

\#4 \#1 OR \#2 OR \#3.

\#5 China OR Chinese OR Taiwan OR "Hong kong" OR Hongkong OR Macau OR Macao OR Beijing OR Shanghai OR Tianjin OR Chongqing OR "Inner Mongolia" OR Tibet OR Guangxi OR Sinkiang OR Ningxia OR Xinjiang OR Hebei OR Shanxi OR Liaoning OR Jilin OR Heilongjiang OR Jiangsu OR Zhejiang OR Anhui OR Fujian OR Jiangxi OR Shandong OR Henan OR Hubei OR Hunan OR Guangdong OR Hainan OR Sichuan OR Guizhou OR Yunnan OR Shaanxi OR Gansu OR Qinghai 110277. \#6 \#4 and \#5. 
4. CNKI: (SU\%=(幽门螺杆菌+幽门螺旋杆菌 +幽门螺旋菌)^感染+hp感染+HP阳性+螺旋菌阳 性+螺杆菌阳性+螺旋菌感染+螺杆菌感染 OR TKA=(幽门螺杆菌+幽门螺旋杆菌+幽门螺旋 菌)^感染+hp感染+HP阳性+螺旋菌阳性+螺杆菌 阳性+螺旋菌感染+螺杆菌感染) AND (SU\%=随 机+盲法+双盲+单盲 + 三盲+交叉+RCT OR $T K A=$ 随机 + 盲法 + 双盲 + 单盲 + 三盲 + 交叉 $+\mathrm{RCT}$ ).

5. Wangfang: 主题:((("幽门螺杆菌" OR "幽门 螺旋杆菌" OR "幽门螺旋菌") AND "感染") OR "hp感染" OR "HP阳性" OR "螺旋菌阳性" OR "螺杆菌阳性" OR "螺旋菌感染" OR "螺杆菌感 染") and 主题:("随机" OR "盲法" OR "双盲" OR "单盲" OR "三盲" OR "交叉" OR "RCT").CBM

6. CBM: (("幽门螺杆菌"[不加权:扩展] OR "幽 门螺杆菌"[常用字段:智能] OR "幽门螺旋杆菌 "[常用字段:智能] OR "幽门螺旋菌"[常用字段: 智能]) AND "感染"[常用字段:智能]) OR ( "hp 感染"[常用字段:智能] OR "HP阳性"[常用字段: 智能] OR "螺旋菌阳性"[常用字段: 智能] OR "螺 杆菌阳性"[常用字段:智能] OR "螺旋菌感染"[常 用字段:智能] OR "螺杆菌感染"[常用字段:智 能]) AND ("临床对照试验"[不加权:扩展] OR " 临床对照试验(主题)"[不加权:扩展] OR "随机对 照试验"[不加权:扩展] OR "随机对照试验(主 题)"[不加权:扩展] OR "非随机对照试验(主 题)"[不加权:扩展] OR "随机"[常用字段:智能] OR "盲法"[常用字段:智能] OR "双盲"[常用字 段:智能] OR "单盲"[常用字段:智能] OR "三盲 "[常用字段:智能] OR "交叉"[常用字段:智能] OR "RCT"[常用字段:智能]).

Participant or population: Chinese Helicobacter pylori (Hp) patients. Diagnosis of $\mathrm{Hp}$ infection will be based on one positive results on one of established techniques including Rapid Urease Test (RUT), Histology (with specific staining), Urease Breath Test (C13 or C14), or any molecular methods. Serological measurements are not acceptable. There will be no limitation on age and gender. Either newly diagnosed patients or treatment failure patients will be included.
Treatment failure patients was defined as a persistent $\mathrm{Hp}$ infection at least 4 weeks after completion of a first-line treatment. China includes mainland, Hong Kong, Macao, and Taiwan.

Intervention: 1. Bismuth-containing quadruple therapy. 2. Triple therapy. 3 . Sequential therapy. 4. Concomitant therapy/ Non-bismuth containing quadruple therapy. 5. Amoxicillin high dose dual therapy. 6. Vonoprazan-Bismuthcontaining quadruple therapy. 7. Placebo. 8. Different treatment duration of quadruple therapy, such as 7 days, 10 days and 14 day. Characteristic components are: antibiotic (Clarithromycin, Cefuoxime, amoxicillin, Levofloxacin, Furazolidone, Tetracycline, Metronidazol), acid suppression drug (omeprazole, lansoprazole, rabeprazole, pantoprazole, esomepraxole, Ilaprazole), Vonoprazan, Bismuth.

Comparator: 1. Bismuth-containing quadruple therapy. 2. Triple therapy. 3. Sequential therapy. 4. Concomitant therapy/ Non-bismuth containing quadruple therapy. 5. Amoxicillin high dose dual therapy. 6. Vonoprazan-Bismuthcontaining quadruple therapy. 7. Placebo. 8. Different treatment duration of quadruple therapy, such as 7 days, 10 days and 14 day. Characteristic components are: antibiotic (Clarithromycin, Cefuoxime, amoxicillin, Levofloxacin, Furazolidone, Tetracycline, Metronidazol), acid suppression drug (omeprazole, lansoprazole, rabeprazole, pantoprazole, esomepraxole, Ilaprazole), Vonoprazan, Bismuth.

\section{Study designs to be included: RCTs.}

Eligibility criteria: We included Chinese Helicobacter pylori $(\mathrm{Hp})$ patients who diagnosed of $\mathrm{Hp}$ infection based on one positive results on one of established techniques including Rapid Urease Test (RUT), Histology (with specific staining), Urease Breath Test (C13 or C14), or any molecular methods. Serological measurements are not acceptable. There were no limitation on age and gender. 
Either newly diagnosed patients or treatment failure patients were included. Treatment failure patients were defined as a persistent $\mathrm{Hp}$ infection at least 4 weeks after completion of a first-line treatment. China includes mainland, Hong Kong, Macao, and Taiwan.

Information sources: We conducted a systematic search in the databases of PubMed, EMBASE, The Cochrane Library, CNKI. Wangfang and CBM. The electronic search will be supplemented by handsearching of reference lists of relevant systematic reviews at full-text screening stage. This is intended to identify any additional published or unpublished material (grey literature) not retrieved by the electronic search.

Main outcome(s): Primary outcome: Eradication rate at end of study (last follow-up timepoint), defined by original studies. Secondary outcome: 1. Adverse events, including total adverse events and specific adverse events. 2. Compliance, defined by original studies. If intention-totreat (ITT) population is available in original studies, we will select outcome data from ITT population. If ITT population is not available, we will use data derived from per-protocol (PP) population.

Quality assessment / Risk of bias analysis: Assessment will be performed according to criteria for assessment of risk of bias recommended by the Cochrane 2019 (e.g. the Cochrane Risk of bias tool for RCTs).

Strategy of data synthesis: Network metaanalysis will be applied for this systematic review. For direct treatment comparisons, we will conduct pairwise meta-analyses by synthesizing studies that compared the same interventions using a random-effects model that contained two or more studies. We will calculate comparative effect sizes as risk ratios (RRs) with their 95\% confidence intervals (Cls). To evaluate the presence of clinical heterogeneity, we will generate descriptive statistics for the population characteristics across all eligible studies that compared each pair of interventions. We will assess the presence of clinical heterogeneity within pairwise comparisons by comparing these characteristics. The substantial statistical heterogeneity will be defined as $12>50 \%$, coupled with $P$ value from $Q$ test less than 0.1 . When there is a substantial statistical heterogeneity, we will explore the sources of heterogeneity. If the source of heterogeneity was identified, we will conduct subgroup analysis to present the result. If the source of heterogeneity was not identified, we will narratively describe the results from individual studies. For a given comparison, say $B$ versus $C$, direct evidence is provided by studies that compare these two treatments directly as in standard direct comparisons metaanalysis. In addition, indirect evidence for $A$ versus $B$ can be provided if studies that compare $A$ versus $C$ and $A$ versus $C$ are analysed jointly. Indirect and direct comparisons meta-analysis aim to combine the direct and indirect evidence into a single effect size and thus may help to increase the precision of the comparison, while randomisation is respected. To check the assumption of consistency in the indirect and direct, we plan to use the design-by-treatment interaction model as fully explained in Higgins 2012.

Subgroup analysis: Subgroup analysis will be applied only for primary outcome. To explore the effect of the age, we plan to perform a subgroup analysis dividing the RCTs into 3 groups, children (aged younger than 18), adults (aged between 18 to 60) and the old (age older than 60). To explore the effect of the different period, we plan to perform a subgroup analysis dividing the RCTs into 2 groups according to recruiting time before and after 2010 (The Fourth National Consensus on the Treatment of Helicobacter Pylori Infection).

Sensitivity analysis: We will not perform sensitivity analysis.

Language: No limited.

Country(ies) involved: China.

Keywords: Helicobacter Pylori (Hp); Chinese population; Network meta-analysis 
Contributions of each author:

Author 1 - Rong Lin have involved in the conception of this study, designed it, collected data and will analyze the data and participate in data interpretation.

Email: selinalin35@hotmail.com

Author 2 - Jing $\mathrm{Li}$ have involved in the conception of this study, designed it, collected data and will analyze the data and participate in data interpretation.

Email: lijing01_2007@126.com

Author 3 - Huiying Shi have involved in the conception of this study, and will participate in data interpretation.

Email: shihuiying23@hotmail.com

Author 4 - Feng Zhou have involved in the conception of this study, designed it, and will participate in data interpretation.

Email: fang.zhou1@takeda.com

Author 5 - Li Xie have involved in the conception of this study, designed it, and will participate in data interpretation.

Email: li.xie@takeda.com 